\title{
Iodine and NIR Dye Labeled Biodegradable Polymer Nanoparticles for Dual CT/NIR Imaging
}

\author{
Vishwas N. Joshi ${ }^{1}$ \\ 1. Anvesha Labs, Setauket, NY, USA
}

The various imaging approaches have their own benefits and drawbacks with respect to their temporal and spatial resolution, sensitivity, penetration depth (or field of view) and, cost. These modalities cover the electromagnetic spectrum ranging from radio waves (MRI) via near-infrared (NIR) down to X-ray (X-ray computed tomography or CT) and gamma radiation (nuclear tomographic imaging). One effective method of synergistically exploiting the advantages of the various modalities is their combined use [1].

Optical imaging provides high sensitivity, low consumption and facile operation. The biological systems strongly absorb in the ultraviolet and visible region but are "transparent" in the near infrared or NIR (750$1400 \mathrm{~nm}$ ) ranges. Therefore, NIR probes have been developed and commercialized for biological imaging. However, NIR fluorescence (NIRF) suffers from its own shortcomings that include low spatial resolution and limited penetration depth, even at red and NIR wavelengths [2]. While CT is a cost effective and efficient imaging modality that provides high spatial resolution and unlimited penetration, the commonly used iodinebased CT imaging agents (ICAs) clear rapidly through kidneys and by extravascular diffusion enabling only short imaging times with very limited or no target-specific imaging. Further, they cause adverse reactions in patients with allergies, asthma, kidney diseases and diabetes [3]. The advantageous complementarities of both $\mathrm{CT}$ and NIRF can be realized by combining CT and NIR imaging agents in a single probe. However, the simple combination of the two imaging probes may not be useful unless they both have identical pharmacodynamic properties. Also, the molecular nature of CT and NIR probes prevents target-specific and temporal-imaging. Nanotechnology can address these problems [4]; therefore, we prepared nanoparticle-based agents labeled with both CT and NIR dyes that will stay in the vascular system for a longer time, reduce kidney burden, and enable bi-modal target specific- and temporal-imaging [4].

The amine functionalized $\mathrm{AB}$ diblock polymers of the type mPEG-b-poly(ester- $\left.\mathrm{NH}_{2}\right)$ [where $\mathrm{A}$ is methoxypolyethylene glycol or $\boldsymbol{m P E G}(5-20 \mathrm{kDa})$, and B is functionalized polyester bearing pendant amine groups or poly (ester- $\left.\mathrm{NH}_{2}\right)$ (5-30 kDa)], were prepared via ring opening polymerization (ROM) of 1,3dioxolane-2,4-dione derivative bearing a carboxybenzyl-protected pendant amine group using $\mathrm{mPEG}$ as an initiator and dimethylaminopyridine (DMAP) as catalyst; the pendant amino groups were regenerated along the polyester backbone following deprotection under acidic conditions [5]. The iodine (I) and NIR dye Cy-7 dual-labeled polymer was prepared by reacting commercial N-hydroxysuccinimide-derivative of Cy-7 and freshly prepared acid chloride derivative of diatrizoic acid in the presence of triethyl amine (TEA), Scheme I. The polymer was purified by LH-60 gel chromatography with dimethylformamide as eluent. The dual-labeled nanoparticle probes were prepared by modified spontaneous emulsification solvent diffusion method [6]. Additional triblock polymers of the type $\mathrm{BAB}$ and $\mathrm{ABC}$, where $\mathrm{C}$ is poly(lactide), and their dual-labeled nanoparticles were also prepared.

The freeze-dried dual-labeled nanoparticles exhibited very good contrast in the microCT (Figure 1A \& 1B; VivaCT 40, SCANCO Medical AG, Brüttisellen, Switzerland). The new nanoparticle agents also formed polymersomes because of hydrophilic $\mathrm{mPEG}$ and hydrophobic polyester (with pendant I and Cy-7 agents on the polyester backbone) blocks (Figure 1C - 1E). The I/Cy-7 dual-labeled nanoparticles could be detected down to 50 millimoles per liter of iodine and 10 micromoles per liter of Cy-7 respectively (Figure 1F \& 1G; 
MARS photon-counting micro-CT). The X-ray contrast (in Hounsfield units), increases linearly with increasing concentrations of the I/Cy-7 dual-labeled nanoparticle agents as expected (Figure 1H).

The new nanoparticle-based probes are composed of both FDA approved (PLGA type polyester and clinical ICA) and generally regarded as safe (GRAS) components (mPEG); therefore, their transition from pre-clinal to clinical settings will be quick and easy.

\section{References:}

[1] A. Louie, Chem. Rev., 110 (2010), p. 3146.

[2] B. Zhu, and E. M. Sevick-Muraca, Br. J. Radiol., 88 (2015), p. 20140547.

[3] H. Lusic et al, Chem. Rev., 113 (2013), p. 1641.

[4] J. E. Rosen et al, J. Nanomed. \& Nanotech., 2 (2011), p. 1000115.

[5] O. T. du Boullay et al, J. Am. Chem. Soc., 128 (2006), p. 16442.

[6] H. Murakami et al, Int. J. Pharmaceutics, 187 (1999), p. 143.

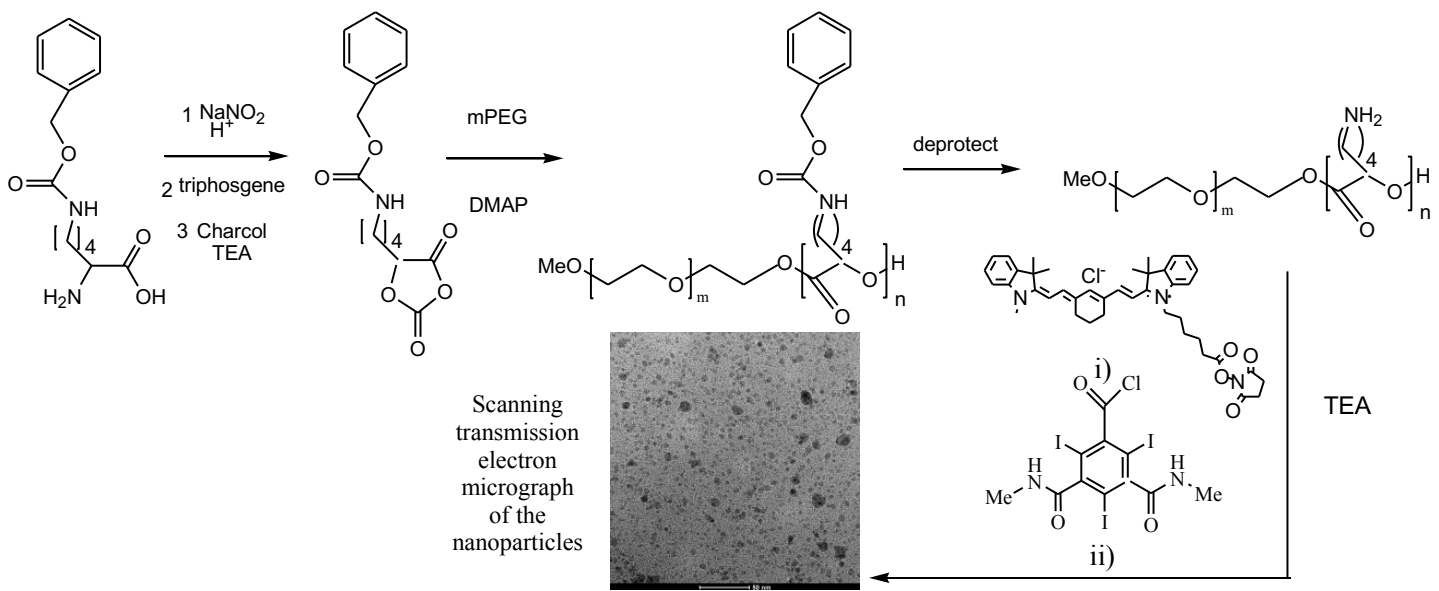

Scheme I: Preparation of iodine and NIR dye dual-labeled mPEG-b-polyester nanoparticles.

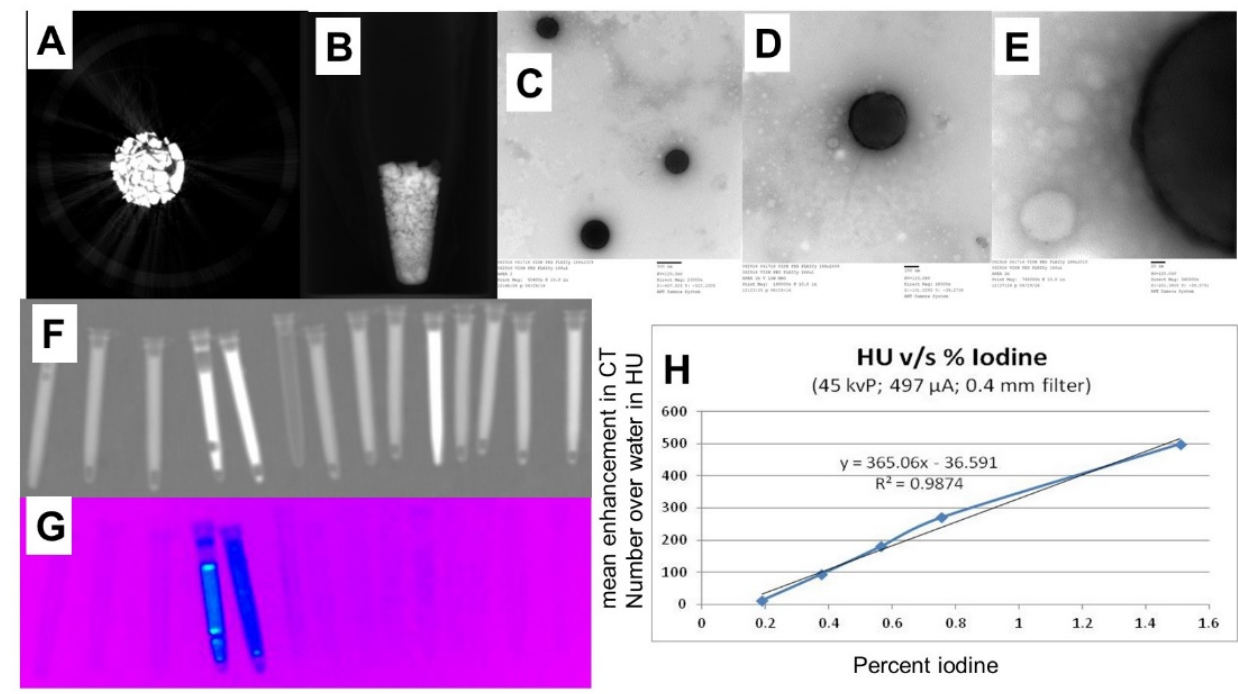

Figure 1: A \& B) microCT slices through freeze-dried lysine-based mPEG-b-polylactide cross-linked to amidotrizoic acid and Cy-7 (mPEG-b-polylactide-I/Cy-7); C, D \& E) TEM of mPEG-b-polylactide-I/Cy-7 polymersomes; F) planar X-ray; G) fluorescence of serially diluted iodine standards and mPEG-b-polylactideI/Cy-7 solutions; and $\mathbf{H}$ ) mean enhancement number over water in $\mathrm{HU}$ for the new probes (from $\mathbf{F}$ ). 